\title{
Article \\ TDE-Based Adaptive Integral Sliding Mode Control of Space Manipulator for Space-Debris Active Removal
}

\author{
Zhibin Zhang ${ }^{1}$, Xinhong $\mathrm{Li}^{1}$, Xun Wang ${ }^{1, *}$, Xin Zhou ${ }^{2}$, Jiping An ${ }^{1}\left(\mathbb{D}\right.$ and Yanyan $\mathrm{Li}^{1}$ \\ 1 Department of Aerospace Science and Technology, Space Engineering University, Beijing 101416, China; \\ zhangzhibinseu@163.com (Z.Z.); 13366159269@189.cn (X.L.); ajp112233@alumni.sjtu.edu.cn (J.A.); \\ yanyanli0212@163.com (Y.L.) \\ 2 Beijing Institute of Remote Sensing information, Beijing 100192, China; zx284061096@163.com \\ * Correspondence: wxhello123@163.com
}

check for updates

Citation: Zhang, Z.; Li, X.; Wang, X.; Zhou, X.; An, J.; Li, Y. TDE-Based Adaptive Integral Sliding Mode Control of Space Manipulator for Space-Debris Active Removal. Aerospace 2022, 9, 105. https:// doi.org/10.3390/aerospace9020105

Academic Editor: George Z. H. Zhu

Received: 28 October 2021

Accepted: 24 January 2022

Published: 16 February 2022

Publisher's Note: MDPI stays neutral with regard to jurisdictional claims in published maps and institutional affiliations.

Copyright: (C) 2022 by the authors. Licensee MDPI, Basel, Switzerland. This article is an open access article distributed under the terms and conditions of the Creative Commons Attribution (CC BY) license (https:// creativecommons.org/licenses/by/ $4.0 /)$.

\begin{abstract}
The safe and dependable removal of large-scale space debris has been a long-standing challenge that is critical to the safety of spacecraft and astronauts. In the process of capturing and deorbiting space debris, the space manipulator must achieve extremely high control and precision. However, strong couplings, model uncertainties, and various inevitable unknown disturbances cause many difficulties in coordinated control of the space manipulator. To solve this challenge, this study examines the stabilization control of a space manipulator after capturing non-cooperative large-scale space debris and presents an adaptive integral sliding mode control (AISMC) scheme with time-delay estimation (TDE). The coupling term and lumped uncertainty are estimated by TDE technology, which eliminates the requirement of prior knowledge. Adaptive sliding mode control (ASMC) is used as desired injecting dynamics to compensate TDE errors, and a PID-type integral sliding mode surface is designed to reduce steady-state errors. The Lyapunov criterion is used to prove the global stability of the controller. Simulation results show that the controller has high tracking accuracy and strong robustness.
\end{abstract}

Keywords: active debris removal; space manipulator; postcapture control; TDE; AISMC

\section{Introduction}

Space debris has huge kinetic energy, posing a major threat to spacecraft in orbit and astronauts' space activities. If active removal is not implemented, frequent collisions would occur, and the amount of space debris will rapidly and uncontrollably increase, posing a huge threat to the space system's safety [1]. Active debris removal (ADR) is an effective space debris mitigation approach that can prevent secondary debris formation at its source and avoid the Kessler syndrome [2,3]. ADR mainly targets large debris, such as failed spacecraft and rocket bodies. Through appropriate means, LEO debris can be burned into the atmosphere [4], while GEO debris can be lifted into the grave orbit, thus enabling clean-up of the space environment.

The capture and deorbit removal technology using a robotic arm is an extremely promising ADR scheme. In comparison to soft capture technologies such as tether-net capture [5], it avoids entanglement and critical oscillation and provides higher controllability and safety, albeit at the cost of increased control accuracy [6]. Normally, the capturing operation by space robotics can be divided into three phases: approach, contact, and postcapture phase [7]. For the safety of the following operation tasks, it is desired to stabilize the combined system as soon as possible in the postcapture phase [8]. The key to ADR is to accomplish force/torque control of space robots to catch and decelerate debris targets. The challenges are estimating and processing system uncertainties, such as external disturbances, model parameter uncertainty, measurement errors, unknown and uncertain inertia $[9,10]$. 
The postcapture control of space robots has been extensively studied. Xu proposed a method for dual-arm space robots to capture moving targets by combining visual prediction and trajectory planning, and successfully captured moving targets in free-floating and base-controlled modes [11]. Luo used angular momentum allocation to achieve postcapture stability of the space robot system, which can successfully handle the speed restriction problem with variable joint performances [7]. Wang proposed an optimal unrolling strategy for the postcapture stage, considering the roll time and control torque, and designed a coordination control scheme to track the designed reference path [12]. Huang studied the postcapture attitude takeover control problem. Considering the uncertainty of inertial parameters, a novel reconfigurable control law was designed by command filtering adaptive back-stepping control to guarantee the system performance and global asymptotic [13]. Liu studied the target unrolling scheme of the flexible base space robot after capture and designed a coordination controller to track the planned trajectory [14]. However, the aforementioned study did not take into account the impact of parameter uncertainties and unknown disturbances at the same time.

The estimation and compensation of the manipulator system's uncertainties and external disturbances is a critical issue to resolve [15-17]. Fuzzy logic [18] and neural network [19] can provide accurate estimates of uncertainties. However, using approximate principles in a space manipulator system for estimation necessitates a large amount of calculation, which makes it challenging to apply in practical applications. Lumped uncertainty can also be estimated by disturbance observer or extended state observer [20], but the design of observer adaptive gain is often complicated. All of the approaches above can provide reliable estimates of unknown quantities, but they all require prior knowledge of dynamic uncertainty, namely, the upper limit must be known in advance [21]. Unfortunately, since these values are dependent on a variety of factors such as load fluctuation, external disturbances, dynamic uncertainties, and so on, it is difficult to presuppose these values. In addition, time delay estimation (TDE) is also an effective method to deal with uncertainty [22,23]. TDE can estimate uncertainty accurately through a simple structure without any prior knowledge of the uncertainty bounds, but when the uncertainty changes sharply in a short time, or there are hard nonlinearities such as saturation and Coulomb friction, the TDE errors will increase significantly, leading to serious degradation of control performance. At the same time, TDE errors are inevitable owing to sampling time constraints, hence TDE errors must be compensated.

To compensate for the abovementioned TDE errors and achieve increased accuracy and robustness, TDE is frequently combined with robust control strategies such as ASMC [24,25], TSMC [26,27], etc. Baek proposed an ASMC scheme based on TDE technology and adopted an adaptive law, which achieved good tracking performance in the case of small jitter. However, the adaptive law would appear singularity when the sliding variable crosses zero, and the tracking accuracy needs to be improved [28]. Bae took the fuzzy sliding mode control scheme as the auxiliary control scheme of time-delay control (TDC), which has less chattering [29]. The adaptive robust TDC proposed by Roy provides robustness against the TDE errors, and the evaluation of switching gains does not depend on any threshold values, which alleviates the over- and underestimation problems of switching gains [30]. Lee proposed an adaptive integral sliding mode control (AISMC) with TDE, and the dynamic injection part uses adaptive gain dynamics to achieve applicable high tracking accuracy, but the finite-time stability of the system cannot be guaranteed [31]. Much of the preceding research solely focused on system stability and did not investigate systems' steady-state performance. When an ASMC based on TDE technology tracks any trajectory, external disturbances may introduce certain steady-state errors that are difficult to fix by parameter adaptation, preventing the ASMC from achieving satisfactory performance.

According to the discussion above, a novel TDE-based AISMC is proposed to achieve improved tracking accuracy and reduced chattering. The proposed controller integrates AISMC as injecting desired dynamics into TDC and inherits the advantages of both TDE based ASMC and TDC. The PID-type integral sliding surface can reduce the steady-state 
errors and improve system stability. The gain dynamics is adaptively updated when the sliding variable changes, allowing the controller to accommodate parameter variation induced by robot attitude changes. The sign function term in the traditional SMC is retained to ensure the robustness of the system, and the chattering effect is effectively reduced through the adaptation of the switching gain. The main contribution lies in the following.

1. The PID-type integral sliding mode surface is designed to effectively reduce the steady-state errors and ensure the robustness of motion throughout the state space.

2. The asymptotic performance of the controller is improved by the exponential reaching law and the sliding mode surface can be reached in finite time.

3. A new adaptive law is utilized to update the gain parameters in response to the system's dynamic changes, improving the closed-loop system's tracking accuracy and stability.

The rest of this paper is structured as follows. In Section 2, we describe the problem to be solved and model the postcapture combined spacecraft. In Section 3, a model-free TDE-based AISMC method is designed for controlled systems containing uncertainties and disturbances, and its closed-loop stability is demonstrated. Our controller is applied to the free-flying space manipulator (FFSM) consisting of a five-DOF robotic arm coupled with a six-DOF base, and simulation results are presented in Section 4 . The research conclusions are given in Section 5 .

\section{Problem Formulation and Preliminaries}

As illustrated in Figure 1, the mission scenario explored in this study involves a service FFSM being deployed to capture a failed spacecraft in LEO. In the postcapture stage, the space manipulator's end-effector securely grasps the target to form a combined system, causing a significant shift in inertial parameters. The entire system is composed of a sixDOF spacecraft base and an n-DOF manipulator. Compared with the space manipulator without load, the difficulties of postcapture control lie in: (1) Space debris is usually a noncooperative target, so it is difficult to identify the parameters accurately. (2) The base is subject to greater interference and requires greater control force and torque for stabilization.

(3) Higher control accuracy is required to avoid collisions.

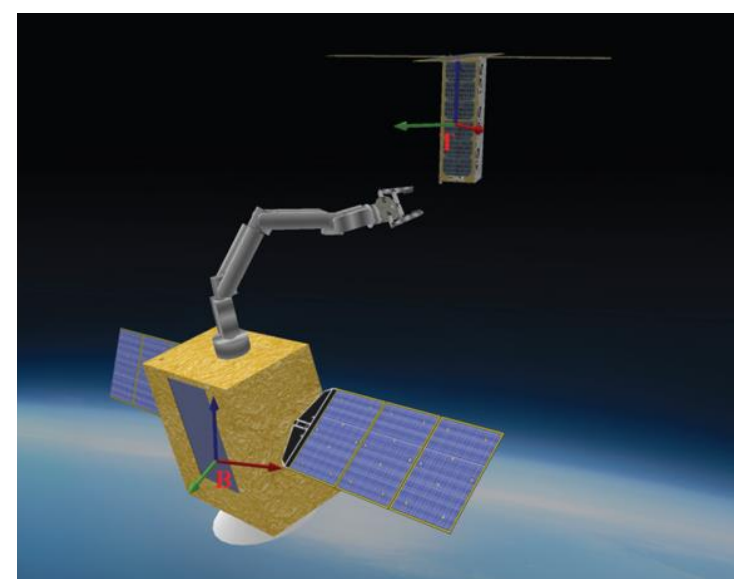

Figure 1. Artistic illustration of a mission scenario to capture a target using a space manipulator.

The dynamic equation of space manipulator established by Lagrange method can be expressed as [32,33]

$$
\underbrace{\left[\begin{array}{cc}
H_{\mathrm{B}} & H_{\mathrm{BM}} \\
H_{\mathrm{BM}}{ }^{T} & H_{\mathrm{M}}
\end{array}\right]}_{H} \underbrace{\left[\begin{array}{c}
\ddot{x}_{\mathrm{B}} \\
\ddot{\theta}_{\mathrm{M}}
\end{array}\right]}_{\ddot{q}}+\underbrace{\left[\begin{array}{c}
C_{\mathrm{B}} \\
C_{\mathrm{M}}
\end{array}\right]}_{C}+\underbrace{\left[\begin{array}{c}
\tau_{d \mathrm{~B}} \\
\tau_{d \mathrm{M}}
\end{array}\right]}_{\tau_{d}}=\underbrace{\left[\begin{array}{c}
F_{\mathrm{B}} \\
\tau_{\mathrm{M}}
\end{array}\right]}_{\tau}
$$


where $q=\left[\begin{array}{ll}x_{\mathrm{B}}^{T} & \theta_{\mathrm{M}}^{T}\end{array}\right]^{T} \in \Re^{6+n}$ is the generalized coordinate, $x_{\mathrm{B}}=\left[r_{\mathrm{B}}^{T}, \theta_{\mathrm{B}}^{T}\right]^{T}, r_{\mathrm{B}}$ and $\theta_{\mathrm{B}}$ are the position and attitude angle of the base, $\theta_{\mathbf{M}}=\left[\theta_{1}, \theta_{2}, \cdots, \theta_{n}\right]$ is the joint angle of the robotic arm; $H \in \Re^{(6+n) \times(6+n)}$ is the inertial matrix of the system, $H_{\mathrm{B}} \in \Re^{6 \times 6}, H_{\mathrm{M}} \in \Re^{n \times n}$ and $H_{\mathrm{BM}} \in \Re^{6 \times n}$ are the inertial matrix of the spacecraft, the robotic manipulator, and the coupling dynamics between the spacecraft and the manipulator respectively; $C \in \Re^{6+n}$ is the generalized Coriolis and centrifugal force term of the system, $C_{B}$ and $C_{M}$ are the Coriolis and centrifugal force terms that correspond to the base and manipulator, respectively; $\tau_{d} \in \Re^{6+n}$ stands for unknown external disturbances; $\tau \in \Re^{6+n}$ is the generalized driving force and torque of the system, including the driving force and torque $F_{\mathrm{B}}$ when the base is in attitude and orbit control, and the joint driving torque $\tau_{\mathrm{M}}$.

To facilitate the design of the controller, the dynamic model Equation (1) can be rewritten as

$$
\begin{aligned}
\ddot{q} & =-H^{-1}\left(C+\tau_{d}\right)+\left(H^{-1}-\hat{H}^{-1}\right) \tau+\hat{H}^{-1} \tau \\
& =N+\hat{H}^{-1} \tau
\end{aligned}
$$

where $\hat{H}$ is the nominal part of $H$, and $N=-H^{-1}\left(C+\tau_{d}\right)+\left(H^{-1}-\hat{H}^{-1}\right) \tau$ is the lumped uncertainty containing model uncertainties and external disturbances.

\section{TDE-Based AISMC}

For the large-scale debris capture operation scenario considered in this study, the controller is designed to track desired trajectories to enable the combination to obtain a stable attitude and configuration. This is achieved by simultaneously controlling the movement of the spacecraft base and the robotic arm in free-flying mode. To address the inherent nonlinearity, parameter uncertainties, and external disturbances of the system, the control scheme combining TDE and SMC is widely used. However, the SMC based on TDE technology still has large steady-state errors that are difficult to reduce via parameter adaptation. Therefore, a PID-type integral sliding mode surface is used in this section to reduce the steady-state errors. On this basis, we present a TDE-based AISMC with exponential reaching law and demonstrate its stability.

\subsection{Controller Design}

The control objective is to make the generalized coordinate $q$ follow the reference coordinate $q_{d}$, which means that the tracking error $e=q_{d}-q$ is suppressed as much as possible. The sliding mode control system's response is composed of the approaching motion of the moving point and the sliding mode motion on the sliding mode surface. The choice of sliding surface has a great influence on the system's tracking effect. Ordinary sliding mode control may cause steady-state errors due to external disturbances when tracking any trajectory. To achieve the control goal and reduce the steady-state errors, the PID-type sliding variable is defined as follows

$$
s=e+K_{D} \dot{e}+K_{P} \int_{0}^{t} e(\tau) d \tau
$$

where $K_{D}$ and $K_{P}$ are positive diagonal gain matrices. The above PID-type sliding variable is used to eliminate the reaching phase and to provide an ideal sliding motion $(\dot{s}=s=0)$.

With Equations (2) and (3), the AISMC controller with exponential approach law can be designed as follows:

$$
\tau=-\hat{H} N+\hat{H}\left(\ddot{q}_{d}+K_{D} \dot{e}+K_{P} e+K_{s} s+G \text { sgns }\right)
$$

where $K_{s}=\operatorname{diag}\left(K_{s 1}, K_{s 2}, \cdots, K_{s n}\right) \in \Re^{n \times n}$ is a time-varying positive diagonal gain matrix, known as the gain dynamics, which determines the convergence speed and convergence performance of the controller. $G=\operatorname{diag}\left(G_{1}, G_{2}, \cdots, G_{n}\right) \in \Re^{n \times n}$ is a time-varying positive diagonal gain matrix, called switching gain, used to compensate for lumped uncertainty. 
Since $N$ contains unknown terms which cannot be obtained directly, an estimated value $\hat{N}$ is obtained from the delayed sampling of $N$ by using TDE [22,23]. In other words, we have

$$
\hat{N}=N(t-L)=\ddot{q}(t-L)-\hat{H}^{-1} \tau(t-L)
$$

where $L$ is the delay constant, usually taken as the actual sampling time to ensure that $L$ is small enough. Substituting Equation (5) into Equation (4) and omitting the time variable $t$, then the TDE-based AISMC is obtained.

$$
\tau=\underbrace{-\hat{H} \ddot{q}_{t-L}+\tau_{t-L}}_{\mathrm{TDE}}+\underbrace{\hat{H}\left(\ddot{q}_{d}+K_{D} \dot{e}+K_{P} e+K_{s} s+G \text { sgns }\right)}_{\mathrm{AISMC}}
$$

A block diagram for the proposed TDE-based AISMC is illustrated in Figure 2. Acceleration $\ddot{q}$ is obtained by differential algorithm, $\ddot{q}=\left(\dot{q}-\dot{q}_{t-L}\right) / L$, to avoid direct measurement of acceleration.

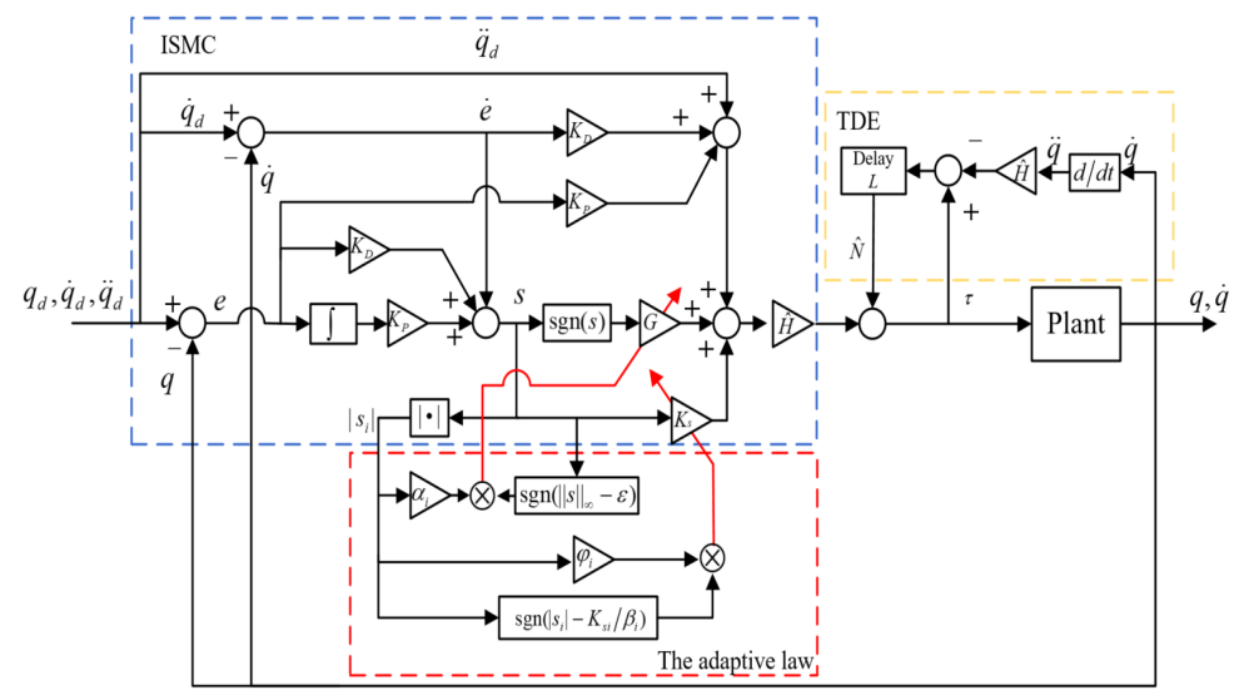

Figure 2. Block diagram of the proposed TDE-based AISMC.

Substitute Equation (6) into Equation (2), replace the sliding variable $s$ with Equation (3), the closed-loop dynamics becomes

$$
\dot{s}=\ddot{e}+K_{D} \dot{e}+K_{P} e=-\tilde{N}-K_{s} s-G \operatorname{sgn} s
$$

where $\widetilde{N}=N-\hat{N}$ is called TDE errors. If $N$ can be accurately estimated, that is, $\widetilde{N}=0$, then the system has exponential approaching law, and the approaching speed can be adjusted by selecting appropriate parameters $K_{s}$ and $G$. If the sampling time $L$ is sufficiently small, the estimation $\hat{N}$ in Equation (5) can be as close to $N$ as possible.

The bounded condition of TDE errors is given in [30,34], if $\hat{H}$ satisfies the following condition

$$
\left\|E-H^{-1} \hat{H}\right\|_{2}<1
$$

for all $t \geq 0$, the TDE errors are bounded by constant, i.e., $|\widetilde{N}| \leq N^{*}$, then the stability of the system is guaranteed. In practice, information such as the system's nominal mass parameters and the maximum allowable payload is always available, so it is always possible to obtain a matrix $\hat{H}$ that satisfies the condition Equation (8).

In reality, due to the inherent measurement noise and limited sampling period [35], TDE errors cannot be completely avoided. The robust term Gsgns can compensate the TDE errors, and also serve as the equal-velocity approaching law term so that the velocity is nonzero when $s$ approaches zero, ensuring the finite time arrival of the approaching motion [36]. To ensure fast approaching and restrain chattering at the same time, $K_{s}$ should 
be increased while $G$ should be appropriately decreased. Especially when the error is small, a small $G$ should be used to avoid chattering.

The gain dynamics is updated by the following adaptive law:

$$
\dot{K}_{s i}=\alpha_{i}\left|s_{i}\right| \operatorname{sgn}\left(\left|s_{i}\right|-K_{s i} / \beta_{i}\right)
$$

where $\alpha_{i}$ is the adjustable positive gain adapted to the speed, $\beta_{i}$ is the normalization factor related to the tracking accuracy, satisfying $\beta_{i}<N^{*} /\|s\|_{\infty}$. When $\left|s_{i}\right|>K_{s i} / \beta_{i}$, the gain dynamics $K_{s i}$ increases, which reduces the TDE errors and tracking errors. If $\left|s_{i}\right|<K_{s i} / \beta_{i}$, the gain dynamics $K_{s i}$ is reduced to prevent the gains from being too high when the errors are acceptable. $K_{s i} / \beta_{i}$ determines the tracking accuracy, and the normalization factor $\beta_{i}$ is used to adjust the size of $K_{s i}$ relative to $\left|s_{i}\right|$. The item $K_{s i} / \beta_{i}$ can be regarded as the acceptance layer of tracking accuracy. The smaller the acceptance layer, the higher the tracking accuracy. By adjusting $\beta_{i}$, the tracking accuracy can be ensured within an appropriate range.

The switching gain is updated by the following adaptive law:

$$
\dot{G}_{i}=\left\{\begin{array}{cl}
\varphi_{i}\left|s_{i}\right| \operatorname{sgn}\left(\| s||_{\infty}-\varepsilon\right) & \text { if } G_{i}>0 \\
\varphi_{i} \varepsilon & \text { if } G_{i}=0
\end{array}\right.
$$

where, $\varphi_{i}$ is the adjustable positive gain that adapts to the speed, and $\varepsilon$ is a small positive number, which determines the boundary of the increase or decrease of the switching gain $G_{i}$.

The proposed adaptive law Equation (10) doesn't require boundary information of uncertainties and disturbances and is activated when the sliding variable $s$ deviates from zero. To elaborate, for $G_{i}>0$, the adaptive law has two different forms according to the output of the sign function: $\|s\|_{\infty} \geq \varepsilon$ and $\|s\|_{\infty}<\varepsilon$. When $\|s\|_{\infty} \geq \varepsilon$, the switching gain $G_{i}$ increases until $\|s\|_{\infty}<\varepsilon$. With the increase of switching gain, the sliding variable $s$ approaches the sliding manifold more quickly. Once the sliding variable enters the vicinity of the sliding manifold, i.e., $\|s\|_{\infty}<\varepsilon$, the switching gain $G_{i}$ decreases while the sliding variable stays in the vicinity of the sliding manifold. The parameter $\varepsilon$ plays a key role in the trade-off between tracking ability and chattering suppression. If $\varepsilon$ is too small, the adaptive speed is slow and there is obvious chattering. On the contrary, if $\varepsilon$ is too large, the tracking performance of the AISMC scheme is poor. In order to avoid system instability caused by excessive chattering, the range of $G$ is limited to $G \leq N^{*}$.

The softening effect of the well-designed adaptive gain ensures the smooth movement of the system's trajectory and prevents the damage of mechanical components. As the injecting desired dynamics, AISMC can effectively suppress the TDE errors, ensure system stability while reducing chattering of sliding mode control. Without knowing the upper boundaries of the disturbances, the TDE technique employed in the study simply assumes that they are bounded. If the disturbances are infinite, then the system is uncontrollable.

Figure 2 is the schematic diagram of the proposed controller. The blue box represents the ISMC part, the red box represents the adaptive law, and the yellow box represents TDE

\subsection{Stability Analysis}

Before showing the UUB property of the proposed control law (6), we introduce a Lemma that will be helpful in the proof of the main results.

Lemma 1. For the system (2) controlled by Equation (6), if the TDE errors are bounded, i.e., $\widetilde{N}<N^{*}$, then the gain dynamics is upper bounded, i.e., $K_{s i}<N^{*}$. 
Proof of Lemma 1. According to the definition, the range of the parameter $\beta_{i}$ is $\beta_{i}<N^{*} /\|s \mid\|_{\infty}$. If $\left|s_{i}\right|>K_{s i} / \beta_{i}$, then

$$
K_{s i}<\left|s_{i}\right| \beta_{i} \leq\|s\|_{\infty} \beta_{i}<\|s\|_{\infty} \frac{N^{*}}{\|s\|_{\infty}}=N^{*}
$$

When $\left|s_{i}\right|_{\infty}<K_{s i} / \beta_{i}$, according to the adaptive law Equation (9), there is $\dot{K}_{s i}<0$, then the gain $K_{s i}$ is reduced. Therefore, $K_{s i}<N^{*}$ for all $t \geq 0$.

The stability of the system can be proved by the Lyapunov stability criterion. The range of the sliding variable $s_{i}$ is assumed to be in $\forall\left|s_{i}\right|>\max \left(K_{s i} / \beta_{i}, \varepsilon\right)$. The Lyapunov function is defined as follows:

$$
V=\frac{1}{2} s^{T} s+\frac{1}{2} \sum_{i=1}^{n} \frac{1}{\alpha_{i}}\left(K_{s i}-N^{*}\right)^{2}+\frac{1}{2} \sum_{i=1}^{n} \frac{1}{\varphi_{i}}\left(G_{i}-N^{*}\right)^{2}
$$

with time derivative as

$$
\dot{V}=s^{T} \dot{s}+\sum_{i=1}^{n} \frac{1}{\alpha_{i}}\left(K_{s i}-N^{*}\right) \dot{K}_{s i}+\sum_{i=1}^{n} \frac{1}{\varphi_{i}}\left(G_{i}-N^{*}\right) \dot{G}_{i}
$$

From Equations (7), (9) and (10), Equation (13) becomes

$$
\begin{aligned}
\dot{V} & =s^{T}\left(-\widetilde{N}-K_{s} s-G s g n s\right)+\sum_{i=1}^{n}\left(K_{s i}-N^{*}\right) \dot{K}_{s i}+\sum_{i=1}^{n}\left(G_{i}-N^{*}\right) \dot{G}_{i} \\
& \leq-\sum_{i=1}^{n}\left|s_{i}\right| \widetilde{N}_{i}-\sum_{i=1}^{n} K_{s i} s_{i}{ }^{2}-\sum_{i=1}^{n}\left|s_{i}\right| G_{i}+\sum_{i=1}^{n}\left(K_{s i}-N^{*}\right)\left|s_{i}\right|+\sum_{i=1}^{n}\left(G_{i}-N^{*}\right)\left|s_{i}\right| \\
& =-\sum_{i=1}^{n} K_{s i} s_{i}{ }^{2}+\sum_{i=1}^{n}\left|s_{i}\right|\left(-\widetilde{N}_{i}-G_{i}+K_{s i}-N^{*}+G_{i}-N^{*}\right) \\
& =-\sum_{i=1}^{n} K_{s i} s_{i}{ }^{2}+\sum_{i=1}^{n}\left|s_{i}\right|\left(K_{s i}-\widetilde{N}_{i}-2 N^{*}\right) \\
& \leq-\sum_{i=1}^{n} K_{s i} s_{i}{ }^{2}+\sum_{i=1}^{n}\left|s_{i}\right|\left(K_{s i}-N^{*}\right) \\
& \leq-\sum_{i=1}^{n} K_{s i} s_{i}{ }^{2}
\end{aligned}
$$

The gain dynamics $K_{s i}>0$, then $\dot{V} \leq 0$, the sliding variable $s$ will enter the range of $\left|s_{i}\right| \leq \max \left(K_{s i} / \beta_{i}, \varepsilon\right)$ in finite time. Since $\dot{V}$ is not guaranteed to be nonpositive in this vicinity of the sliding manifold, the sliding variable $s$ may move in and out of this vicinity repeatedly. Once the sliding variable $s$ leaves the region $\left|s_{i}\right| \leq \max \left(K_{s i} / \beta_{i}, \varepsilon\right), \dot{V}$ becomes negative again, which steers it back toward the sliding manifold.

The first time when the sliding variable $s$ enters the region $\left|s_{i}\right| \leq \max \left(K_{s i} / \beta_{i}, \varepsilon\right)$, there is $\left|s_{i}\right| \leq \max \left(N^{*} / \beta_{i}, \varepsilon\right)$. It can be proved that the Lyapunov function Equation (12) is bounded

$$
\frac{1}{2}\|s\|_{2}{ }^{2} \leq V \leq \frac{1}{2} \sum_{i=1}^{n}\left(\max \left(N^{*} / \beta_{i}, \varepsilon\right)\right)^{2}+\frac{1}{2} \sum_{i=1}^{n} \frac{1}{\alpha_{i}}\left(N^{*}\right)^{2}+\frac{1}{2} \sum_{i=1}^{n} \frac{1}{\varphi_{i}}\left(N^{*}\right)^{2}=V^{*}
$$

When the sliding variable $s$ leaves the region $\left|s_{i}\right| \leq \max \left(K_{s i} / \beta_{i}, \varepsilon\right), \dot{V}$ becomes negative again and $V$ decreases immediately. It follows then that we have

$$
\|s\|_{2} \leq \sqrt{2 V^{*}}=\sqrt{\sum_{i=1}^{n}\left(\max \left(N^{*} / \beta_{i}, \varepsilon\right)\right)^{2}+\sum_{i=1}^{n} \frac{1}{\alpha_{i}}\left(N^{*}\right)^{2}+\sum_{i=1}^{n} \frac{1}{\varphi_{i}}\left(N^{*}\right)^{2}}
$$

This means that the sliding variable $s$ is UUB, and Equation (16) guarantees that the fluctuation of the sliding variable $s$ in the vicinity of the sliding manifold is upper-bounded. 


\subsection{Comparison Schemes}

To gain additional insight, the scheme Equation (6) proposed in this study is compared with the original TDC scheme [22], an existing TDE-based ASMC scheme [28], and an existing TDE-based AISMC scheme [31].

The original TDC scheme in [22] is expressed as

$$
\tau=\underbrace{-\hat{H} \ddot{q}_{t-L}+\tau_{t-L}}_{\mathrm{TDE}}+\underbrace{\hat{H}\left(\ddot{q}_{d}+K_{D} \dot{e}+K_{P} e\right)}_{\text {Injecting desired dynamics }}
$$

The existing TDE-based ASMC scheme in [28] is expressed as

$$
\tau=\underbrace{-\hat{H} \ddot{q}_{t-L}+\tau_{t-L}}_{\mathrm{TDE}}+\underbrace{\hat{H}\left(\ddot{q}_{d}+K_{D} \dot{e}+K_{s} s+G \operatorname{sgn}(s)\right)}_{\mathrm{ASMC}}
$$

with the adaptive gain $G$ updated by

$$
\dot{G}_{i}=\left\{\begin{array}{cc}
\varphi_{i}\left\{\alpha_{i}^{-1}\left|s_{i}\right|\right\}^{\operatorname{sgn}\left(\|s\|_{\infty}-\varepsilon\right)} \operatorname{sgn}\left(\|s\|_{\infty}-\varepsilon\right) & \text { if } G_{i}>0 \\
\varphi_{i} \alpha_{i}{ }^{-1}\left|s_{i}\right| & \text { if } G_{i}=0
\end{array}\right.
$$

The existing TDE-based AISMC scheme in [31] is expressed as

$$
\tau=\underbrace{-\hat{H} \ddot{q}_{t-L}+\tau_{t-L}}_{\mathrm{TDE}}+\underbrace{\hat{H}\left(\ddot{q}_{d}+K_{D} \dot{e}+K_{p} e+K_{s} s\right)}_{\mathrm{AISMC}}
$$

with the gain dynamics $K_{s}$ updated by

$$
\dot{K}_{s i}=\alpha_{i}\left|s_{i}\right| \operatorname{sgn}\left(\left|s_{i}\right|-K_{s i}^{2} / \beta_{i}\right)
$$

Both Equation (6) and Equation (20) have integral sliding mode surfaces. Compared with Equation (20), Equation (6) has one more robust term Gsgn(s) and a simpler adaptive law of $K_{s}$. The robust term ensures the robustness of the system, which is reflected in the proof process of the two controllers. The disadvantage is that chattering is generated, which cannot be eliminated through the adaptation of the switching gain. As the control group, Equations (17) and (18) have ordinary sliding mode surfaces. Similarly, Equation (18) has one more adaptive robust term than Equation (17). The controller (6) proposed in this research can be regarded as an extended and enhanced version of the other three controllers. In detail, if $G=0$, then Equation (6) degenerates into the controller (20). If $K_{P}=0$, the integral sliding surface becomes an ordinary sliding surface, then Equations (6) and (20) degenerate to Equations (18) and (17) respectively.

\section{Numerical Simulation}

Simulations were conducted in MATLAB and Simulink for the free-flying space manipulator consisting of a five-DOF arm coupled with a six-DOF base. The terminal time is set as $t_{f}=20 \mathrm{~s}$, the simulation step is $d t=0.01 \mathrm{~s}$. The fifth-order polynomial is used to design trajectories for the joints of the arm as well as the spacecraft base [37].

Unknown nonlinear disturbances are common problems in the coordinated control of space manipulators, which usually include environmental perturbations such as atmospheric drag, joint friction, and vibrations generated by solar panels and manipulator links. The combination of trigonometric functions is used to simulate external disturbances in the simulation $[38,39]$.

The relevant parameter settings of the spacecraft and manipulator are shown in Table 1 . The parameters of the proposed controller are carefully tuned and detailed as follows: $K_{D}=100 \operatorname{diag}(1,1, \cdots, 1), K_{P}=100 \operatorname{diag}(2,2,2,1,1, \cdots, 1), G(0)=2 \times 10^{-4} \operatorname{diag}$ $(1,1, \cdots, 1), K_{s}(0)=80 \operatorname{diag}(1,1, \cdots, 1), \alpha=3 \times 10^{4} \operatorname{diag}(1,1,1,6,6,6,3,3, \cdots, 3), \varepsilon=10^{-4}$, $\beta=4 \times 10^{7} \operatorname{diag}(1,1,1,6,6,6,3,3, \cdots, 3), \varphi=10^{-2} \operatorname{diag}(1,1,1,10,10, \cdots, 10), b=10^{5} \operatorname{diag}$ 
$(1,1, \cdots, 1), a=10^{-7} \operatorname{diag}(1,1,1,10,10, \cdots, 10)$. To ensure a fair comparison, apply the above parameters to the other controllers entirely.

Table 1. Initialization of simulation parameters.

\begin{tabular}{|c|c|}
\hline Parameters & Value \\
\hline Mass of the spacecraft base & $m_{\mathrm{B}}=400 \mathrm{~kg}$ \\
\hline Inertia of the spacecraft base & $I_{x x}=I_{y y}=I_{z z}=266 \mathrm{~kg} \cdot \mathrm{m}^{2}$ \\
\hline Mass of the links of the arm & $\begin{array}{c}m_{1}=3 \mathrm{~kg}, m_{2}=10 \mathrm{~kg}, m_{3}=10 \mathrm{~kg}, m_{4}=1.5 \mathrm{~kg} \\
m_{5}=1.5 \mathrm{~kg}\end{array}$ \\
\hline Length of the links of the arm & $\begin{array}{c}l_{1}=0.18 \mathrm{~m}, l_{2}=0.62 \mathrm{~m}, l_{3}=0.62 \mathrm{~m} \\
l_{4}=0.09 \mathrm{~m}, l_{5}=0.09 \mathrm{~m}\end{array}$ \\
\hline Mass of the target & $m_{\mathrm{T}}=100 \mathrm{~kg}$ \\
\hline Inertia of the target & $I_{x x}=I_{y y}=I_{z z}=16.7 \mathrm{~kg} \cdot \mathrm{m}^{2}$ \\
\hline Spacecraft base's initial position & $r_{\mathrm{B} 0}=[0.1,0.3,-0.2]^{T} \mathrm{~m}$ \\
\hline Spacecraft base's initial attitude & $\theta_{\mathrm{B} 0}=[0.11,0.08,-0.1]^{T} \mathrm{rad}$ \\
\hline Arm joints' initial angle & $\theta_{\mathrm{M} 0}=[10,20,60,35,-40]^{T} \pi / 180 \mathrm{rad}$ \\
\hline Spacecraft base's target position & $r_{\mathrm{B} T}=[0,0,0]^{T} \mathrm{~m}$ \\
\hline Spacecraft base's target attitude & $\theta_{\mathrm{B} T}=[0,0,0]^{T} \mathrm{rad}$ \\
\hline Arm joints' target angle & $\theta_{\mathrm{M} T}=[-25,65,50,65,-25]^{T} \pi / 180 \mathrm{rad}$ \\
\hline $\begin{array}{l}\text { Disturbances applied on the } \\
\text { position of base }\end{array}$ & $\tau_{d \mathrm{~B}_{r}}=[0.4 \sin (0.3 t), 0.3 \cos (0.1 t), 0.2 \sin (0.3 t)]^{T}$ \\
\hline $\begin{array}{l}\text { Disturbances applied on the } \\
\text { attitude of base }\end{array}$ & $\tau_{d \mathrm{~B}_{\theta}}=[0.4 \sin (0.3 t), 0.3 \cos (0.1 t), 0.2 \sin (0.3 t)]^{T}$ \\
\hline $\begin{array}{l}\text { Disturbances applied on the } \\
\text { robotic arm }\end{array}$ & $\begin{aligned} \tau_{d \mathrm{M}}= & {[0.1 \sin (1.2 t), 0.08 \sin (0.1 t)+0.1,-0.1 \sin (0.6 t), \cdots} \\
& 0.09 \sin (0.8 t+\pi / 4)-0.1,0.1 \cos (t)+0.1]^{T}\end{aligned}$ \\
\hline
\end{tabular}

To estimate the uncertainties and disturbances more accurately and reduce the TDE errors as much as possible, the sampling time is set as the simulation step $L=d t=0.01 \mathrm{~s}$. Assuming that the parameters of the space manipulator are known, the estimated mass of the target is $\hat{m}_{T}=80 \mathrm{~kg}$, and the estimated moment of inertia is $\hat{I}_{T}=\operatorname{diag}(12,12,12) \mathrm{kg} \cdot \mathrm{m}^{2}$. To obtain a more realistic scenario to analyze the performance of the proposed controller, the velocity measurement error is considered in the simulation.

The trajectory planning results and the tracking trajectories of the proposed controller are shown in Figure 3. Figure $3 a$ is the trajectory tracking result of the end effector in three-dimensional task space, Figure $3 b, c$ are the trajectory tracking results of the base attitude and position respectively, and Figure $3 \mathrm{~d}$ is the tracking result of the joint trajectory of the manipulator. It can be seen that TDE can accurately estimate the uncertainties and disturbances, and the coordinated controller can successfully realize the high-precision stability control of the target and the spacecraft.

To verify the superiority of the PID-type integral sliding mode surface, the proposed control method is compared with the other three control schemes in Section 3.3.

Figures 4 and 5 show the tracking errors and control torques under the four controllers. The subscripts a-c represent the $x, y, z$ components of the base attitude, $d$-f represent the $x$, $\mathrm{y}, \mathrm{z}$ components of base position, $\mathrm{g}-\mathrm{k}$ represent joints $1-5$ of the robotic arm, respectively. The proposed TDE-based AISMC scheme and the other three schemes can suppress TDE errors and achieve high control accuracy. The proposed TDE-based AISMC and the existing AISMC are superior to the ASMC and the traditional TDC in terms of tracking error, indicating that the integral sliding mode surface can effectively eliminate the steady-state errors, and improve the control accuracy by nearly one order of magnitude compared with the ordinary sliding mode surface. Through the pairwise comparison of TDE-based AISMC and AISMC, ASMC and TDC, it can be found that the existence of robust items can ensure the robustness of the system and improve the accuracy of the controller to a certain extent, with the disadvantage of generating some chattering. As can be seen from Figure 5, chattering occurs in TDE based AISMC and ASMC schemes, mainly because when the error is close to zero, the change rate of the error will be relatively large, resulting in the 
sliding variable exceeding the acceptable range of switching gain. The parameter $\varepsilon$ in the adaptive law (10) plays a crucial role in the trade-off between robustness and vibration reduction. When $\varepsilon$ is too small, there is significant chattering in the TDE-based AISMC scheme. Conversely, if $\varepsilon$ is too large, the robust performance and tracking accuracy will be reduced. On the other hand, chattering only occurs locally, indicating that the adaptive update of the switching gain can significantly suppress chattering.

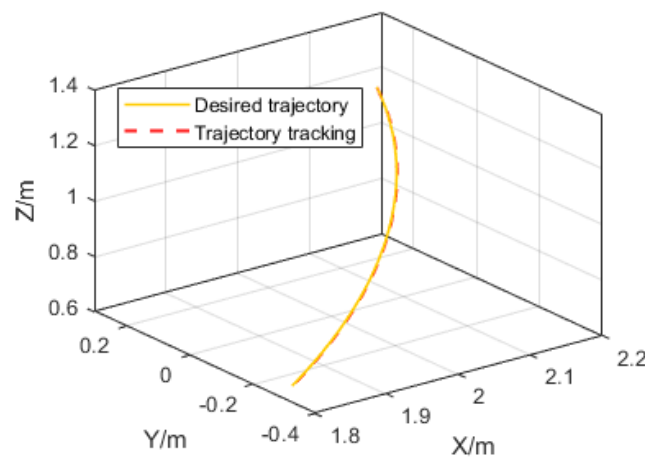

(a)

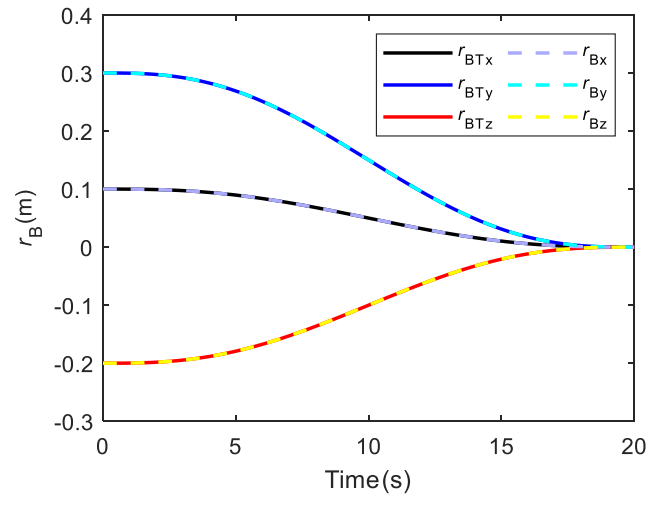

(c)

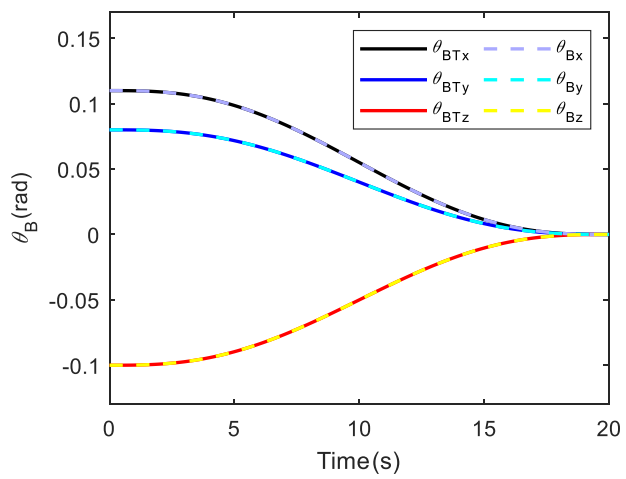

(b)

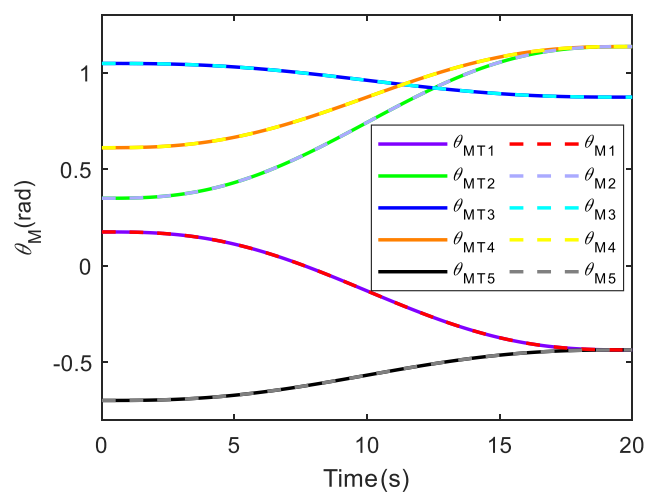

(d)

Figure 3. Trajectory planning results and the tracking trajectories under the proposed controller. (a) Trajectory of the end-effector in task space. (b) Trajectories of the base attitude. (c) Trajectories of the base position. (d) Trajectories of the manipulator joint in joint space.

To evaluate the control performance quantitatively, the integral of time multiplied by the absolute value of the error (ITAE) and the integral of the square value (ISV) of the control input's norm are used. The ITAE is used as a numerical measure of tracking performance for the entire error curve, and the ISV depicts the energy consumption [40]. These are defined as follows:

$$
\begin{aligned}
\text { ITAE } & =\int_{0}^{t_{f}} t\|e(t)\|_{1} d t \\
\mathrm{ISV} & =\int_{0}^{t_{f}}\|\tau(t)\|_{2}{ }^{2} d t
\end{aligned}
$$

Table 2 summarizes the error indicators and control input results of the four controllers under different disturbances. Disturbances $\tau_{d 1}$ are shown in Table 1. $\tau_{d 2}$ have three times the frequency of $\tau_{d 1}$. $\tau_{d 3}$ have three times the amplitude of $\tau_{d 1}$. The symbol * represents the best performance of the four controllers. As can be seen from Table 2, the control accuracy of the proposed controller is the best under different disturbances. Even if the frequency and amplitude of external disturbances increase, the controller can still estimate and compensate for the external disturbances well. The TDE-based AISMC proposed in this study has the highest tracking accuracy (ITAE), but the control input energy consumption (ISV) is slightly larger than the existing AISMC. Similarly, the tracking accuracy of the 
TDE-based ASMC is also higher than that of traditional TDC. These two sets of results show that the robust term can reduce steady-state errors and increase the system's robustness, but also increase the energy consumption of the control input. Because the errors are small and the chattering effect is barely noticeable, compared with the ordinary sliding surface, the integral sliding surface can effectively reduce errors and improve control accuracy. Simulation results verify the effectiveness of the proposed TDE-based AISMC scheme.

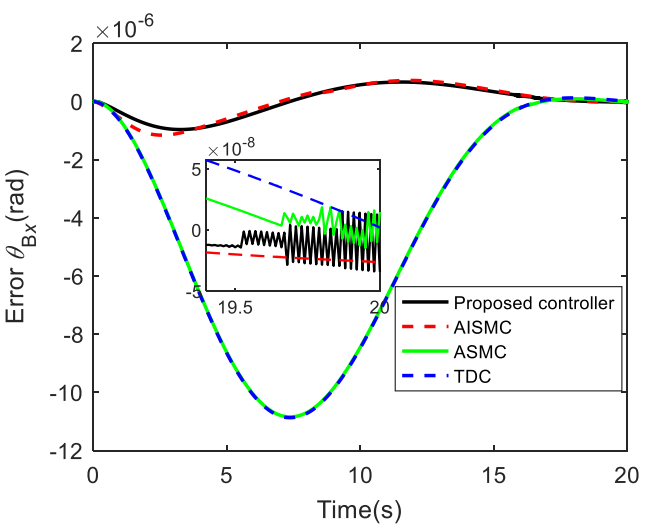

(a)

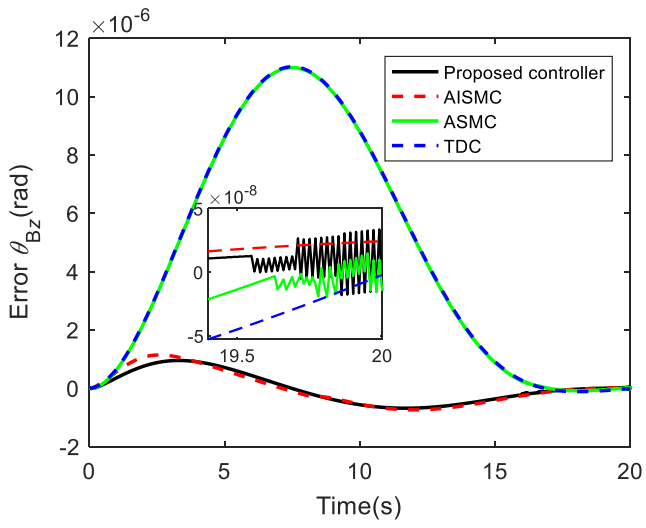

(c)

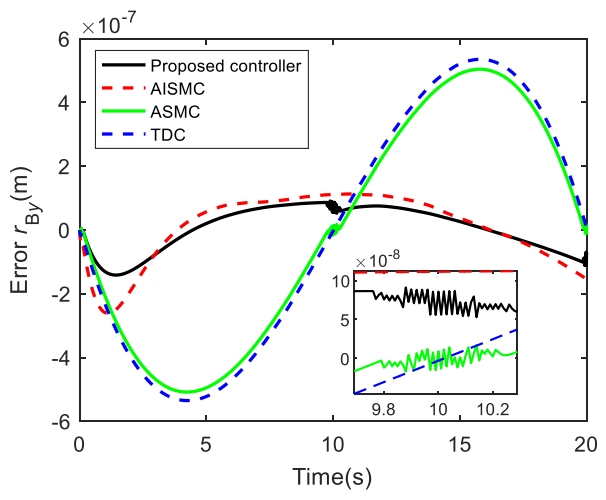

(e)

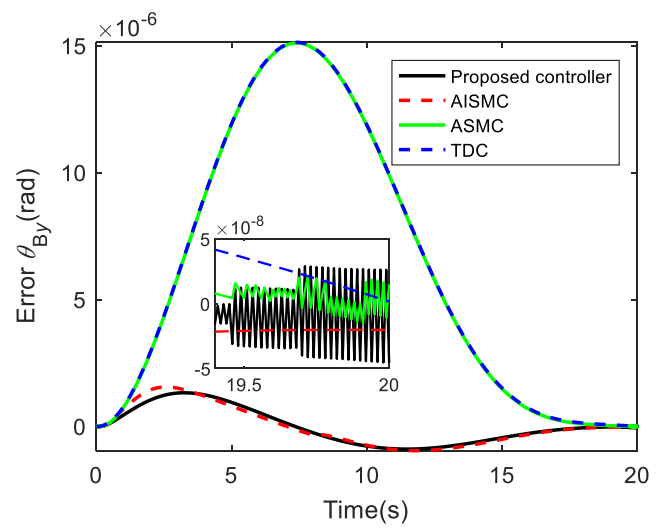

(b)

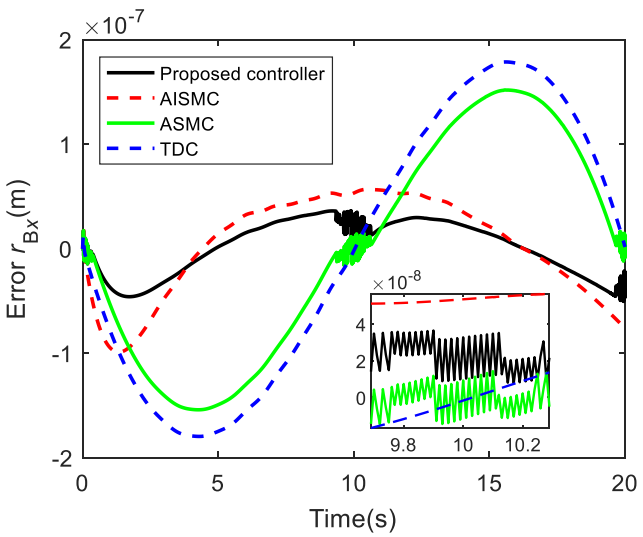

(d)

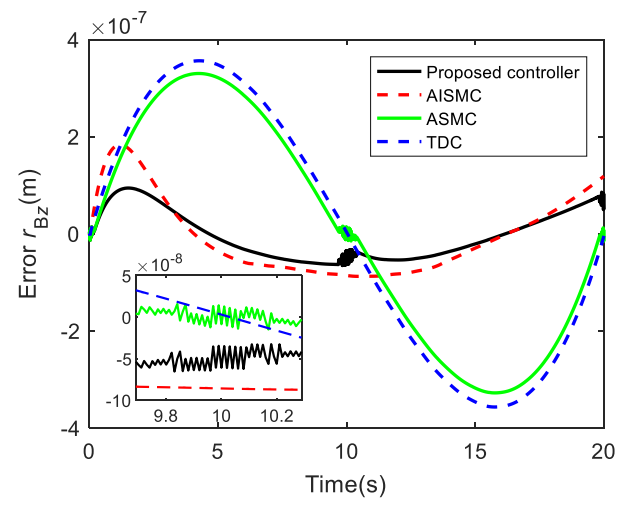

(f)

Figure 4. Cont. 


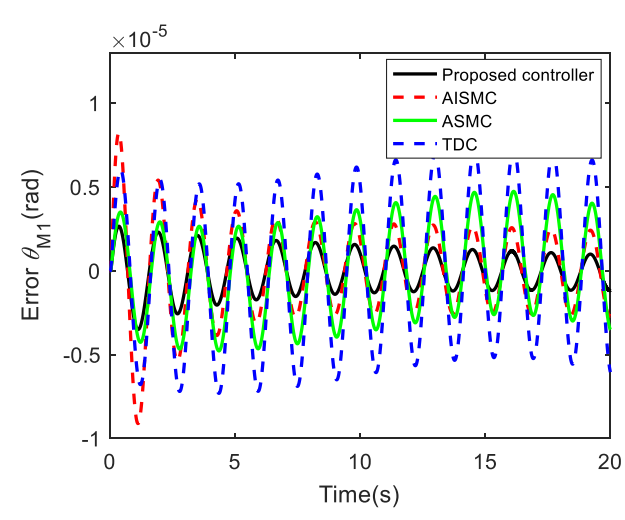

(g)

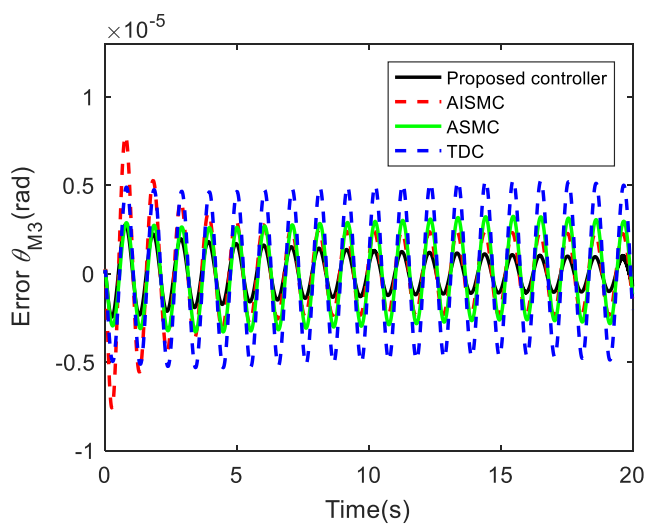

(i)

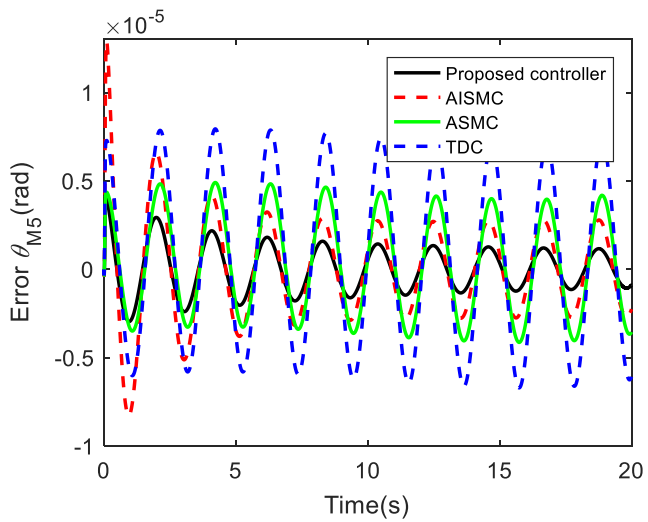

(k)

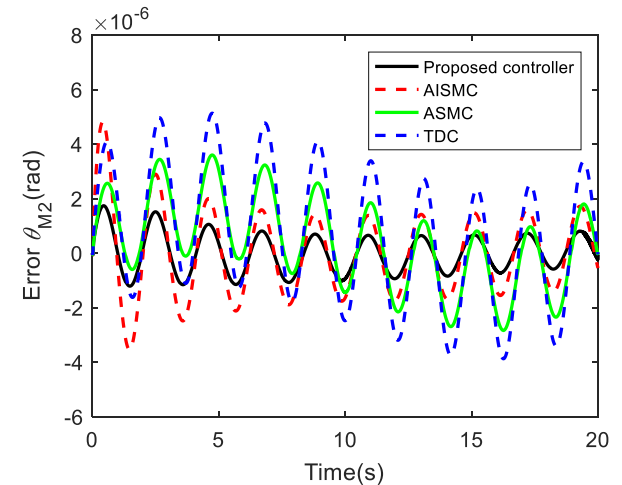

(h)

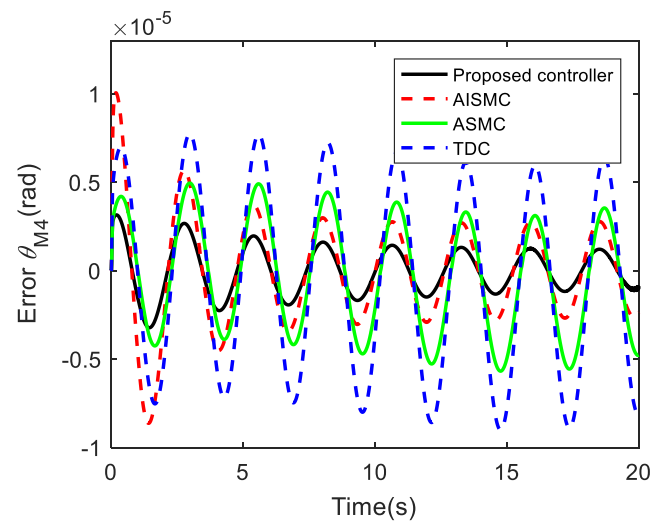

(j)

Figure 4. Comparison of tracking errors under four controllers: (a-c) $x, y, z$ components of base attitude tracking errors. (d-f) $\mathrm{x}, \mathrm{y}, \mathrm{z}$ components of base position tracking errors. (g-k) tracking errors of joints $1-5$, respectively. 


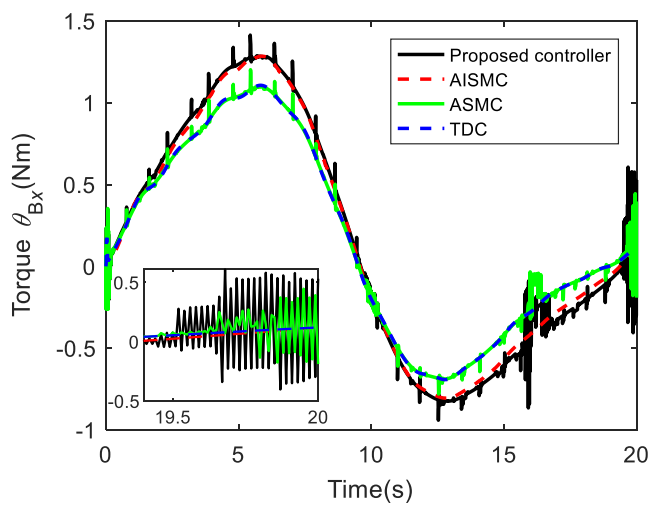

(a)

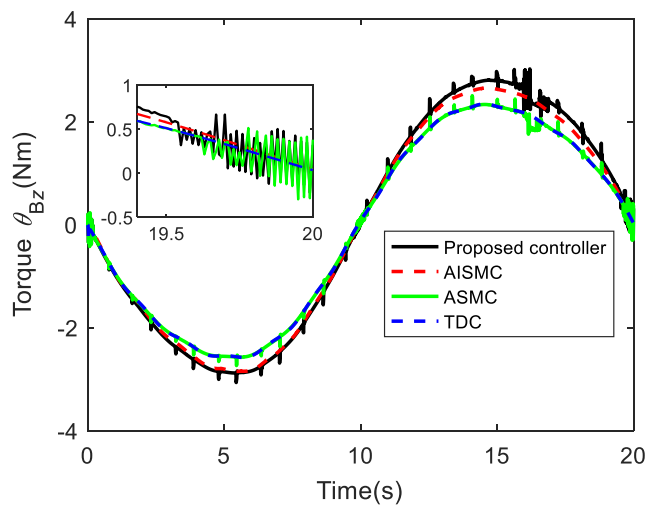

(c)

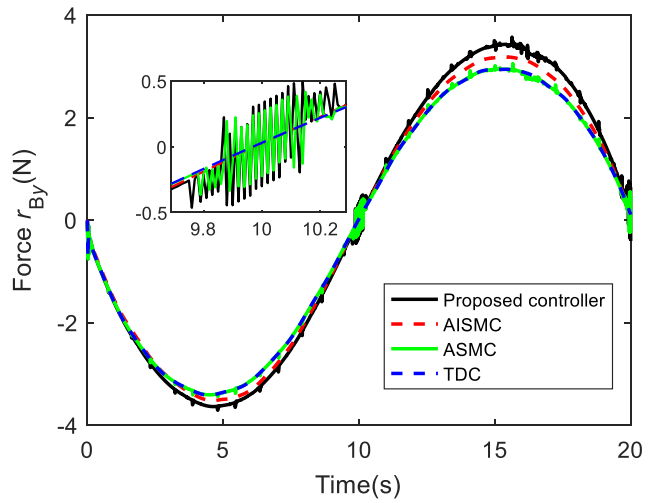

(e)

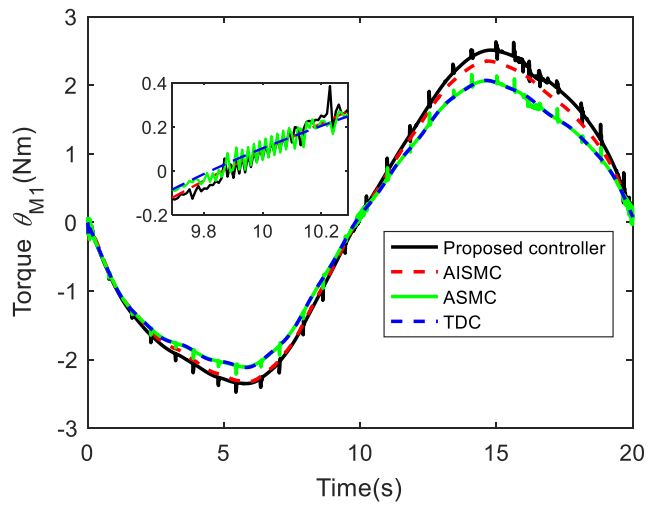

(g)

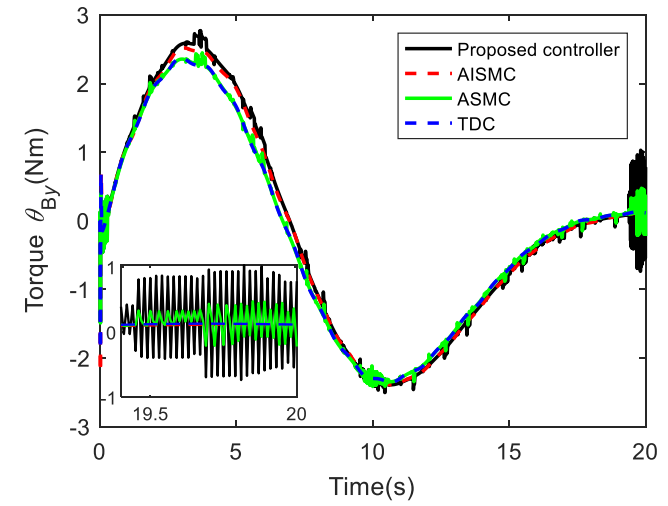

(b)

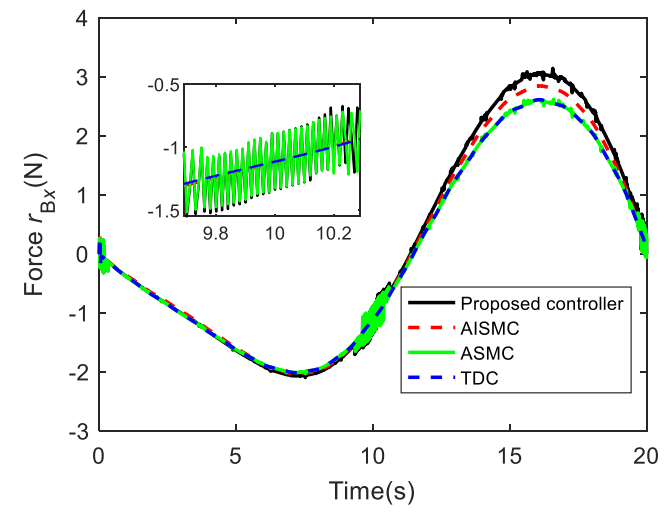

(d)

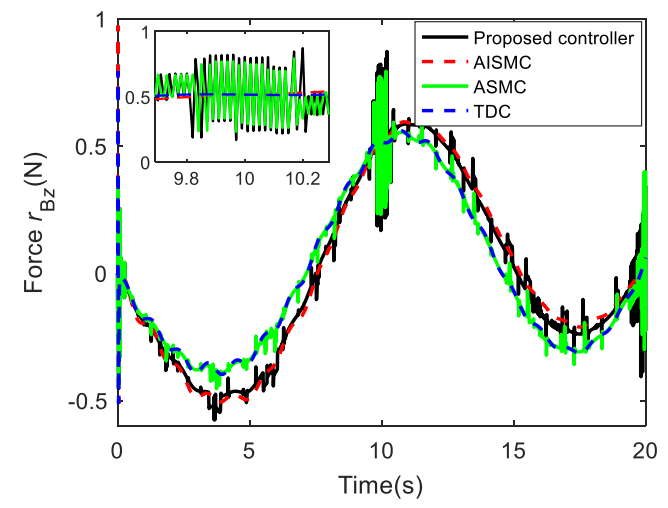

(f)

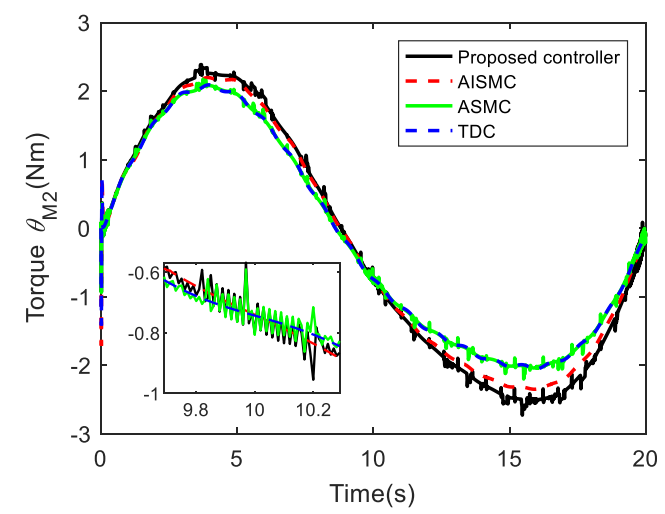

(h)

Figure 5. Cont 


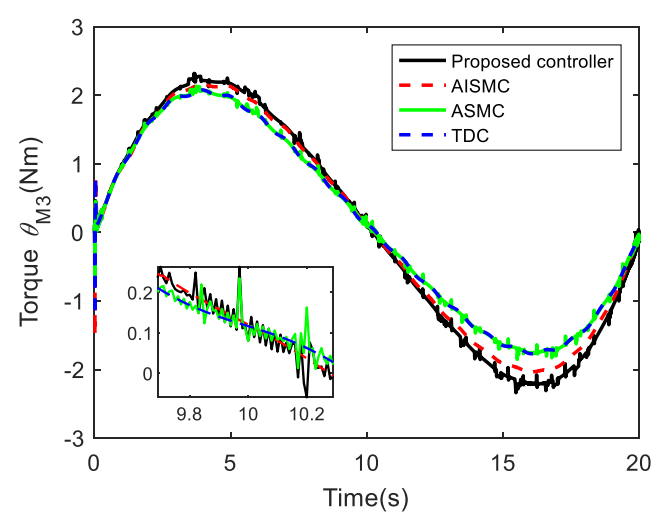

(i)

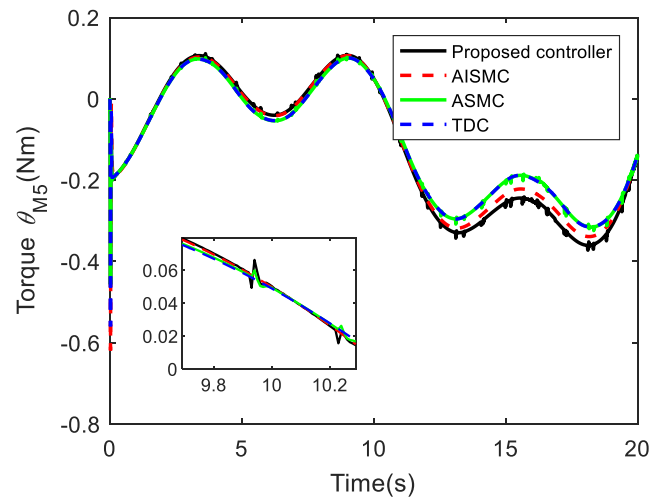

$(\mathbf{k})$

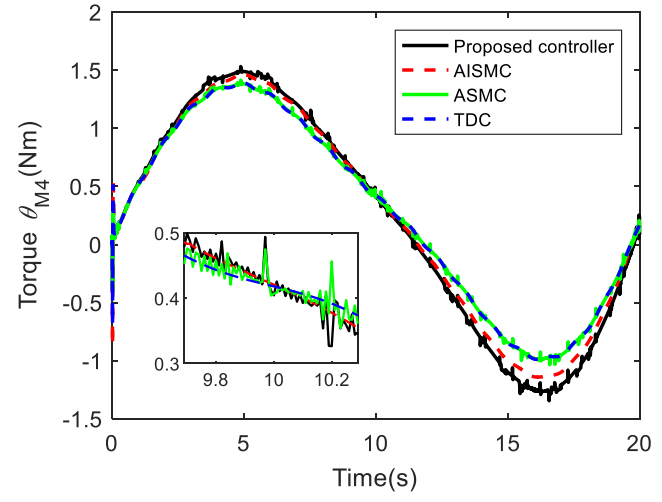

(j)

Figure 5. Comparison of control forces and torques under four controllers: $(\mathbf{a}-\mathbf{c}) \mathrm{x}, \mathrm{y}, \mathrm{z}$ components of base attitude control torques. (d-f) $x, y, z$ components of base position control forces. (g-k) control torques of joints $1-5$, respectively.

Table 2. ITAE and ISV of different control schemes.

\begin{tabular}{cccccc}
\hline Disturbance & Controller & Proposed & AISMC & ASMC & TDC \\
\hline \multirow{2}{*}{$\tau_{d 1}$} & ITAE & $* 1.041 \times 10^{-3}$ & $1.913 \times 10^{-3}$ & $4.725 \times 10^{-3}$ & $6.264 \times 10^{-3}$ \\
& ISV & 393.9 & 387.2 & 377.6 & $* 377.2$ \\
\hline \multirow{2}{*}{$\tau_{d 2}$} & ITAE & $* 1.041 \times 10^{-3}$ & $1.912 \times 10^{-3}$ & $4.726 \times 10^{-3}$ & $6.263 \times 10^{-3}$ \\
& ISV & 368.2 & 361.3 & 351.7 & $* 351.3$ \\
\hline \multirow{2}{*}{$\tau_{d 3}$} & ITAE & $* 1.041 \times 10^{-3}$ & $1.914 \times 10^{-4}$ & $4.726 \times 10^{-3}$ & $6.264 \times 10^{-3}$ \\
& ISV & 489.1 & 482.4 & 472.8 & 472.4 \\
\hline
\end{tabular}

* The best value.

\section{Conclusions}

The problem of coordination control after capturing large space debris with parameter uncertainties and external disturbances was studied in this study, and a robust TDE-based AISMC scheme was proposed. TDE technology was used to compensate for the coupling term and lumped uncertainty, thus eliminating the requirement for prior knowledge. The PID-type integral sliding mode surface can greatly reduce the steady-state errors and increase the system stability at the same time. Compared with previous TDE-based controllers, the proposed method has better tracking performance and stronger robustness. Compared with the single-arm space manipulator, the double-arm space manipulator can greatly enhance the adaptability of the robot to complex tasks, and at the same time improve the utilization efficiency of the working space. In the future, the process of capturing space 
debris by the dual-arm space manipulator will be studied, including target racemization and capture, as well as postcapture control.

Author Contributions: Conceptualization, Z.Z. and X.L.; data curation, X.Z.; formal analysis, Z.Z. and J.A.; funding acquisition, X.L. and X.W.; investigation, J.A.; methodology, Z.Z. and Y.L.; project administration, X.L.; resources, X.Z.; software, X.W.; supervision, X.L. and X.W.; validation, X.Z., J.A. and Y.L.; writing-original draft, Z.Z. and J.A.; writing-review and editing, X.W. and Y.L. All authors have read and agreed to the published version of the manuscript.

Funding: This research was funded by the National Defense Science and Technology Innovation Zone of China, grant number 00205501.

Institutional Review Board Statement: Not applicable.

Informed Consent Statement: Not applicable.

Data Availability Statement: The data presented in this study are available on request from the corresponding author.

Conflicts of Interest: The authors declare no conflict of interest.

\section{References}

1. Liou, J.C. An active debris removal parametric study for LEO environment remediation. Adv. Space Res. 2011, 47, 1865-1876. [CrossRef]

2. Kessler, D.J.; Cour-Palais, B.G. Collision frequency of artificial satellites: The creation of a debris belt. J. Geophys. Res. Space Phys. 1978, 83, 2637-2646. [CrossRef]

3. Kessler, D.J.; Johnson, N.L.; Liou, J.C.; Matney, M. The kessler syndrome: Implications to future space operations. Adv. Astronaut. Sci. 2010, 137, 2010.

4. Zhong, R.; Zhu, Z.H. Dynamics of nanosatellite deorbit by bare electrodynamic tether in low earth orbit. J. Spacecr. Rocket. 2013, 50, 691-700. [CrossRef]

5. Kang, J.; Zhu, Z.H.; Wang, W.; Wang, C.; Li, A. Dynamics and de-spin control of massive target by single tethered space tug. Chin. J. Aeronaut. 2019, 32, 653-659. [CrossRef]

6. Zhao, P.Y.; Liu, J.G.; Wu, C.C. Survey on research and development of on-orbit active debris removal methods. Sci. China Technol. Sci. 2020, 63, 2188-2210. [CrossRef]

7. Luo, J.; Xu, R.; Wang, M. Detumbling and stabilization of a tumbling target using a space manipulator with joint-velocity limits. Adv. Space Res. 2020, 66, 1689-1699. [CrossRef]

8. Flores-Abad, A.; Ma, O.; Pham, K.; Ulrich, S. A review of space robotics technologies for on-orbit servicing. Prog. Aerosp. Sci. 2014, 68, 1-26. [CrossRef]

9. Li, S.; She, Y. Recent advances in contact dynamics and post-capture control for combined spacecraft. Prog. Aerosp. Sci. 2021, 120, 100678. [CrossRef]

10. Liu, C.; Yue, X.; Yang, Z. Are nonfragile controllers always better than fragile controllers in attitude control performance of post-capture flexible spacecraft? Aerosp. Sci. Technol. 2021, 118, 107053. [CrossRef]

11. Yan, L.; Xu, W.; Hu, Z.; Liang, B. Virtual-base modeling and coordinated control of a dual-arm space robot for target capturing and manipulation. Multibody Syst. Dyn. 2019, 45, 431-455. [CrossRef]

12. Wang, M.; Luo, J.; Yuan, J.; Walter, U. Detumbling strategy and coordination control of kinematically redundant space robot after capturing a tumbling target. Nonlinear Dyn. 2018, 92, 1023-1043. [CrossRef]

13. Huang, P.; Lu, Y.; Wang, M.; Meng, Z.; Zhang, Y.; Zhang, F. Postcapture Attitude Takeover Control of a Partially Failed Spacecraft with Parametric Uncertainties. IEEE Trans. Autom. Sci. Eng. 2019, 16, 919-930. [CrossRef]

14. Liu, Y.; Liu, X.; Cai, G.; Chen, J. Trajectory planning and coordination control of a space robot for detumbling a flexible tumbling target in post-capture phase. Multibody Syst Dyn. 2020, 52, 281-311. [CrossRef]

15. Feng, Q.; Zhu, Z.H.; Pan, Q.; Liu, Y. Pose and motion estimation of unknown tumbling spacecraft using stereoscopic vision. Adv. Space Res. 2018, 62, 359-369. [CrossRef]

16. Xie, Z.; Sun, T.; Kwan, T.; Wu, X. Motion control of a space manipulator using fuzzy sliding mode control with reinforcement learning. Acta Astronaut. 2020, 176, 156-172. [CrossRef]

17. Zhang, T.; Yue, X.; Yuan, J. An online one-step method to identify inertial parameters of the base and the target simultaneously for space robots in postcapture. IEEE Access 2020, 8, 189913-189929. [CrossRef]

18. Yang, C.; Jiang, Y.; Na, J.; Li, Z.; Cheng, L.; Su, C.Y. Finite-Time Convergence Adaptive Fuzzy Control for Dual-Arm Robot with Unknown Kinematics and Dynamics. IEEE Trans. Fuzzy Syst. 2019, 27, 574-588. [CrossRef]

19. Yao, Q. Adaptive fuzzy neural network control for a space manipulator in the presence of output constraints and input nonlinearities. Adv. Sp. Res. 2021, 67, 1830-1843. [CrossRef]

20. Zhu, Y.; Qiao, J.; Guo, L. Adaptive sliding mode disturbance observer-based composite control with prescribed performance of space manipulators for target capturing. IEEE Trans. Ind. Electron. 2019, 66, 1973-1983. [CrossRef] 
21. Roy, S.; Baldi, S.; Fridman, L.M. On adaptive sliding mode control without a priori bounded uncertainty. Automatica 2020, 111, 108650. [CrossRef]

22. Hsia, T.C.; Gao, L.S. Robot manipulator control using decentralized linear time-invariant time-delayed joint controllers In Proceedings of the IEEE International Conference on Robotics and Automation, Cincinnati, OH, USA, 13-18 May 1990; pp. 2070-2075. [CrossRef]

23. Hsia, T.C.; Lasky, T.A.; Guo, Z. Robust Independent Joint Controller Design for Industrial Robot Manipulators. IEEE Trans. Ind. Electron. 1991, 38, 21-25. [CrossRef]

24. Li, M.; Chen, Y. Robust Adaptive Sliding Mode Control for Switched Networked Control Systems with Disturbance and Faults. IEEE Trans. Ind. Inform. 2019, 15, 193-204. [CrossRef]

25. Roy, S.; Kar, I.N. Adaptive sliding mode control of a class of nonlinear systems with artificial delay. J. Frankl. Inst. 2017, 354, 8156-8179. [CrossRef]

26. Ahmed, S.; Wang, H.; Tian, Y. Adaptive High-Order Terminal Sliding Mode Control Based on Time Delay Estimation for the Robotic Manipulators with Backlash Hysteresis. IEEE Trans. Syst. Man Cybern. Syst. 2021, 51, 1128-1137. [CrossRef]

27. Chen, G.; Jin, B.; Chen, Y. Nonsingular fast terminal sliding mode posture control for six-legged walking robots with redundant actuation. Mechatronics 2018, 50,1-15. [CrossRef]

28. Baek, J.; Jin, M.; Han, S. A New Adaptive Sliding-Mode Control Scheme for Application to Robot Manipulators. IEEE Trans. Ind. Electron. 2016, 63, 3628-3637. [CrossRef]

29. Bae, H.J.; Jin, M.; Suh, J.; Lee, J.Y.; Chang, P.H.; Ahn, D.S. Control of robot manipulators using time-delay estimation and fuzzy logic systems. J. Electr. Eng. Technol. 2017, 12, 1271-1279. [CrossRef]

30. Roy, S.; Kar, I.N.; Lee, J.; Jin, M. Adaptive-Robust Time-Delay Control for a Class of Uncertain Euler-Lagrange Systems. IEEE Trans. Ind. Electron. 2017, 64, 7109-7119. [CrossRef]

31. Lee, J.; Chang, P.H.; Jin, M. Adaptive Integral Sliding Mode Control With Time-Delay Estimation for Robot Manipulators. IEEE Trans. Ind. Electron. 2017, 64, 6796-6804. [CrossRef]

32. Xie, Z.; Sun, T.; Kwan, T.H.; Mu, Z.; Wu, X. A New Reinforcement Learning Based Adaptive Sliding Mode Control Scheme for Free-Floating Space Robotic Manipulator. IEEE Access 2020, 8, 127048-127064. [CrossRef]

33. Xu, W.; Liang, B.; Xu, Y. Survey of modeling, planning, and ground verification of space robotic systems. Acta Astronaut. 2011, 68, 1629-1649. [CrossRef]

34. Hsia, T.C. Simple robust schemes for cartesian space control of robot manipulators. Int. J. Robot. Autom. 1994, 9, 167-174.

35. Cho, G.R.; Chang, P.H.; Park, S.H.; Jin, M. Robust tracking under nonlinear friction using time-delay control with internal model. IEEE Trans. Control Syst. Technol. 2009, 17, 1406-1414. [CrossRef]

36. Wang, A.; Jia, X.; Dong, S. A new exponential reaching law of sliding mode control to improve performance of permanent magnet synchronous motor. IEEE Trans. Magn. 2013, 49, 2409-2412. [CrossRef]

37. Zhang, X.; Ming, Z. Trajectory Planning and Optimization for a Par4 Parallel Robot Based on Energy Consumption. Appl. Sci. 2019, 9, 2770. [CrossRef]

38. Jia, S.; Shan, J. Finite-Time Trajectory Tracking Control of Space Manipulator under Actuator Saturation. IEEE Trans. Ind. Electron. 2020, 67, 2086-2096. [CrossRef]

39. Qiao, J.; Wu, H.; Yu, X. High-precision attitude tracking control of space manipulator system under multiple disturbances. IEEE Trans. Syst. Man Cybern. Syst. 2021, 51, 4274-4284. [CrossRef]

40. Viveiros, C.; Melício, R.; Igreja, J.M.; Mendes, V.M.F. Performance Assessment of a Wind Turbine Using Benchmark Model: Fuzzy Controllers and Discrete Adaptive LQG. Procedia Technol. 2014, 17, 487-494. [CrossRef] 